\title{
SESAME, un laboratoire d'excellence multidisciplinaire au service des scientifiques du Moyen-Orient
}

\author{
Amor Nadji (1) (amor.nadji@synchrotron-soleil.fr) et Abderrahmane Tadjeddine ${ }^{(2)}$ \\ (abderrahmane.tadjeddine@u-psud.fr) \\ (1) Société civile Synchrotron SOLEIL, L'Orme des Merisiers, Saint-Aubin, BP 48, \\ 91192 Gif-sur-Yvette Cedex \\ (2) Institut de Chimie Physique, UMR8000 CNRS-Université Paris-Sud, 91405 Orsay Cedex
}

\section{Le 16 mai 2017, le centre}

de rayonnement synchrotron de troisième génération

SESAME a été officiellement inauguré par le roi Abdallah II de Jordanie sur le site d'Allan, au nord d'Amman.

\section{C'est l'aboutissement de deux} décennies d'une collaboration internationale ayant pour objectif de créer un centre de recherche scientifique interdisciplinaire ouvert à tous les pays de la région.

\section{Cette inauguration marque}

le début d'une ère nouvelle pour la recherche scientifique, le développement technologique, l'éducation et la coopération au Moyen-Orient.

Nous proposons de retracer ici la genèse et l'état actuel de ce projet créé, à l'image du CERN, sous les auspices de I'UNESCO.

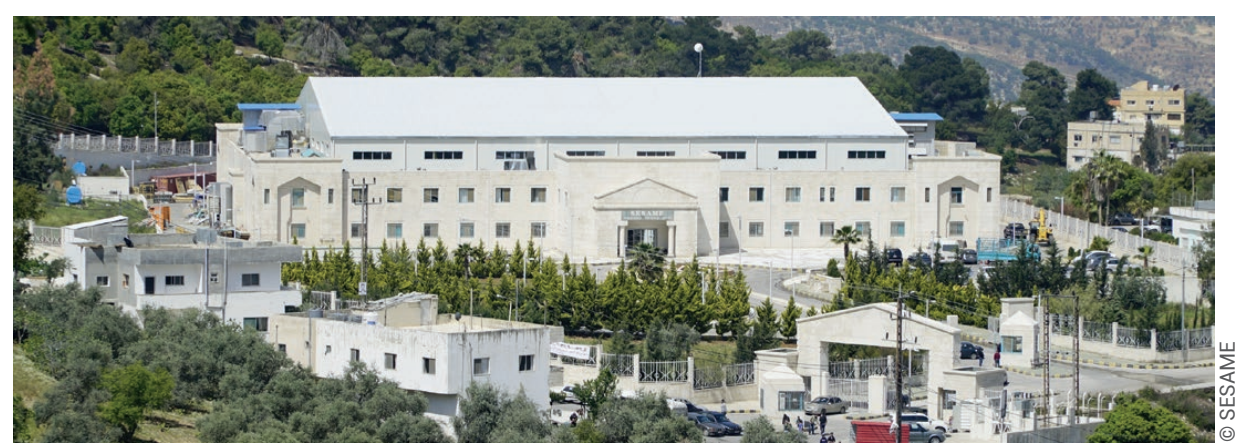

L'objectif du projet SESAME (Synchrotron-light for Experimental Science and Applications in the Middle East) est de promouvoir la science, de favoriser la coopération internationale et la paix, et de fournir aux scientifiques de la région des structures ouvertes d'accueil et de recherche de très haut niveau.

Une telle entreprise implique des volets politiques, financiers, techniques et technologiques, scientifiques et humains, allant du choix du site à la formation des ressources humaines nécessaires, de la construction à l'exploitation du laboratoire, ainsi qu'à la création et au développement d'une communauté multidisciplinaire d'utilisateurs dans la région. Elle a vu le jour grâce au soutien de l'UNESCO et de l'Agence internationale de l'énergie atomique (AIEA), de l'Union européenne et de nombreux pays, ainsi que d'un accompagnement permanent des autres centres de rayonnement synchrotron et du CERN.

Cependant, un centre de rayonnement synchrotron ouvert à une communauté extérieure ne se limite pas à un anneau de stockage et à des lignes de lumière. Il doit offrir des conditions d'accueil, d'hébergement, de rencontres et d'échanges, et s'intégrer dans une dynamique scientifique, technique et de formation impliquant les chercheurs, les ingénieurs, les industriels, les enseignants et les étudiants des pays de la région. C'est une gageure et un défi à relever, que SESAME pourra assumer avec le soutien des institutions qui l'accompagnent depuis sa création, de la communauté scientifique en général, de sociétés savantes telles que la Société Française de Physique, et de nombreuses organisations non gouvernementales.

Après avoir dressé un historique du projet, nous présenterons son évolution technique vers un anneau de troisième génération de 2,5 GeV (gigaélectronvolt), capable de fournir des faisceaux de photons de haute brillance couvrant un domaine spectral allant de l'infrarouge aux rayons X durs, et de sonder les propriétés structurales, électroniques, magnétiques et chimiques de la matière. 


\section{Genèse du projet}

Évoquée dans les années 1980 par le professeur Abdus Salam, prix Nobel de physique, l'idée d'implanter au MoyenOrient un centre de recherche d'excellence ouvert a été approfondie par le groupe de travail CERN-Middle East Scientific Cooperation (MESC), destiné à promouvoir la coopération scientifique régionale, la paix et la solidarité. Le noyau expérimental en serait une source de rayonnement synchrotron, outil essentiel dans de nombreux domaines de la science, permettant de promouvoir des technologies modernes, de favoriser des activités interdisciplinaires et de stimuler des coopérations régionales.

Le passage du rêve à la réalité date de 1997, avec la cession par le gouvernement allemand de l'anneau synchrotron BESSY I de Berlin, en voie d'arrêt d'exploitation et de démantèlement, en vue de son transfert comme source de lumière synchrotron au Moyen-Orient.

Le projet a été lancé en juin 1999 à l'UNESCO, en présence de délégations des pays du Moyen-Orient et d'autres régions, avec la mise en place d'un Conseil intérimaire présidé par Herwig Schopper, ancien directeur général du CERN, et de comités scientifique, technique, financier et de formation coprésidés par des personnalités du Moyen-Orient et des experts du synchrotron, le secrétariat étant provisoirement assuré par l'UNESCO.

Le Conseil intérimaire a organisé dès août 1999 une rencontre de scientifiques des pays potentiellement concernés et de spécialistes du rayonnement synchrotron des pays européens et des USA. Le synchrotron français Lure (Laboratoire pour l'utilisation du rayonnement électromagnétique) était représenté par un directeuradjoint et plusieurs ingénieurs du département des accélérateurs, qui joueront un rôle important dans la conception des sources et des lignes de lumière de SESAME, dans la formation de ses cadres techniques et scientifiques, d'abord au Lure, puis à SOLEIL, aux côtés de collègues des autres centres de rayonnement synchrotron $^{(a)}$. Le transfert de la machine d'Allemagne en Jordanie a été effectué en juin 2002, puis le travail sur le projet a été engagé, sans attendre le choix du site. Une école a été organisée à Amman pour sélectionner puis former des étudiants de la région dans les centres de rayonnement synchrotron européens et des USA, afin de constituer une équipe cohérente d'ingénieurs machine et d'associer des ingénieurs et des techniciens de la région au démantèlement de l'anneau BESSY I, et à sa réinstallation dans le pays hôte.

L'étape suivante a été le choix du site, qui devait remplir les conditions techniques et être localisé dans un pays acceptant d'accueillir tout utilisateur sur l'unique critère de la qualité scientifique de son projet. Parmi les cinq sites candidats, celui d'Allan, dans le campus universitaire d'El Balqa, situé à une trentaine de $\mathrm{km}$ au nord d'Amman en Jordanie, réunissait toutes les conditions, le pays hôte s'engageant à prendre en charge la construction du bâtiment et à apporter un soutien solide au projet. En mai 2002, le Conseil exécutif de l'UNESCO a approuvé la création du Centre sur le site d'Allan.

Les statuts de SESAME, rédigés à l'image de ceux du CERN, ont été déposés à l'UNESCO le 15 avril 2004. Le Conseil intérimaire a alors été remplacé par un Conseil permanent. Un appel à devenir membre de SESAME a été adressé aux pays de la région. Neuf d'entre eux ont répondu positivement : Bahreïn (qui s'est peu impliqué et n'est plus membre depuis 2017), Chypre, l’Égypte, l’Iran, Israël, la Jordanie, le Pakistan, la Palestine et la Turquie. La France, l'Union européenne, l'Allemagne, le Brésil, le Canada, la Chine, l'Espagne, la Grèce, l'Italie, le Japon, le Koweït, le Portugal, la Russie, la Suède, la Suisse et les USA sont observateurs.

Le projet s'est poursuivi sous la direction du Conseil permanent à raison de deux réunions annuelles, sans interférence notable des conditions extérieures et des nombreuses tensions de la région, l'administration de SESAME restant hébergée dans les locaux de l'UNESCO à Amman jusqu'à son installation dans son site propre à Allan en 2008.

Le choix a rapidement évolué vers une source de troisième génération, avec une énergie de 2,5 GeV, impliquant la conception et la construction d'un anneau de stockage spécifique, utilisant BESSY I comme injecteur, et des changements profonds de l'ensemble de l'infrastructure (voir p. 21). Ce choix a entrainé une augmentation conséquente du budget d'investissement, financé principalement par l'Union européenne et certains pays membres de SESAME. Le premier faisceau d'électrons a été délivré en juillet 2009 par le microtron remonté et mis à niveau par l'équipe technique de SESAME, puis injecté dans le nouvel anneau de stockage de SESAME en janvier 2017. Le premier faisceau de photons monochromatique a été extrait le 22 novembre 2017 sur la ligne Spectroscopie d'absorption et de fluorescence X (XAFS/XRS), qui a accueilli ses premiers utilisateurs en juillet 2018.

SESAME a été inauguré le 16 mai 2017 par le roi Abdallah II de Jordanie, en présence de la Directrice générale de l'UNESCO, du Commissaire européen à la Recherche, de la Directrice générale du CERN, de représentants de l'AIEA et de délégations des pays membres et observateurs.

À l'heure actuelle, trois lignes de lumière (XAFS/XRS, Spectromicroscopie infrarouge et Science des matériaux) sont opérationnelles, la maison d'hôtes vient d'être inaugurée et l'installation d'une centrale solaire qui couvrira en partie la consommation électrique de SESAME est achevée.

\section{Source de rayonnement synchrotron}

Le rayonnement synchrotron est constitué d'ondes électromagnétiques émises par les électrons circulant dans des anneaux de stockage à des vitesses proches de celle de la lumière et soumis à une accélération centripète ou à un changement de trajectoire sous l'effet de champs magnétiques. Ses propriétés exceptionnelles - polarisation, gamme spectrale allant du térahertz et de l'infrarouge aux rayons $\mathrm{X}$ durs de plusieurs dizaines de $\mathrm{keV}$, brillance (nombre de photons/seconde/unité d'angle solide/ unité de surface) de un million à un milliard de fois plus intense que le faisceau délivré par les sources de lumière conventionnelles en font un outil multidisciplinaire incontournable de caractérisation de la structure de la matière dans tous ses états et à toutes échelles, justifiant la construction de sources dédiées [1].

La figure 1 représente le schéma-type d'une source de rayonnement synchrotron. Un faisceau d'électrons, émis par un canon à électrons, est accéléré dans un accélérateur linéaire (LINAC) ou circulaire (microtron). Il est ensuite injecté dans un deuxième accélérateur circulaire appelé booster qui porte l'énergie des électrons à la plus haute valeur accessible $(800 \mathrm{MeV}$ pour SESAME). Enfin, le faisceau pénètre dans l'anneau de stockage où les électrons 


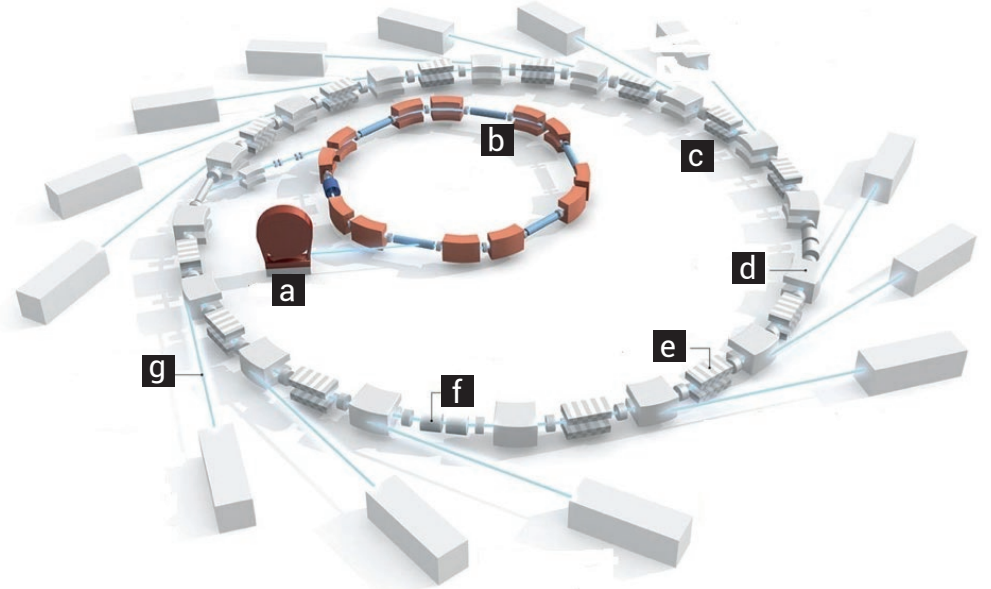

1. Schéma simplifié de la source de rayonnement synchrotron SESAME.

a : cathode fournissant des électrons accélérés par un accélérateur circulaire (microtron)

$\mathrm{b}$ : anneau injecteur (booster)

c : anneau de stockage

$\mathrm{d}$ : aimants de courbure

e : éléments magnétiques d'insertion

$\mathrm{f}$ : cavité radiofréquence

$\mathrm{g}$ : lignes de lumière [2]

sont éventuellement accélérés à leur énergie nominale $(2,5 \mathrm{GeV}$ pour SESAME) et tournent pendant plusieurs heures. Des dispositifs magnétiques, dipôles (ou aimants de courbure), onduleurs ou wigglers (succession périodique d'aimants de polarités alternées), dévient leur trajectoire ou les font osciller. Les électrons ainsi freinés perdent de l'énergie sous forme d'un rayonnement électromagnétique appelé "rayonnement synchrotron ». Ces pertes d'énergie sont exactement compensées à chaque tour par des cavités radiofréquence (RF). Le rayonnement synchrotron produit dans les aimants de courbure et les éléments d'insertion (wigglers ou onduleurs) est sélectionné, conditionné et dirigé par les systèmes optiques des lignes de lumière vers les stations expérimentales [2]. La conception de la source de rayonnement synchrotron est optimisée en fonction de ses applications [3].

\section{Évolution de la machine SESAME : de Bessy I vers une machine de troisième génération à $2,5 \mathrm{GeV}$}

Plusieurs ateliers organisés au MoyenOrient ont montré que le programme scientifique de SESAME devait inclure la biologie moléculaire et structurale, les sciences de l'environnement, l'imagerie X, la microanalyse archéologique, la caractérisation des matériaux et des applications médicales. Ces domaines de recherche nécessitent des photons $\mathrm{X}$ durs allant jusqu'à $20 \mathrm{keV}$, ce qui a impliqué la conception d'un nouvel anneau de stockage utilisant, après une jouvence appropriée, BESSY I comme injecteur. Ainsi, la structure magnétique et les dimensions de l'anneau ont évolué pour intégrer les besoins des utilisateurs et porter les performances de SESAME au niveau de celles des anneaux de troisième génération. Une conception initiale à $2 \mathrm{GeV}$ [4] a été modifiée pour atteindre $2,5 \mathrm{GeV}$, suite à la première réunion des utilisateurs potentiels de SESAME
D1

$$
\text { D1 D3 D4 D5 }
$$

D5 D4 D3 D2

D6

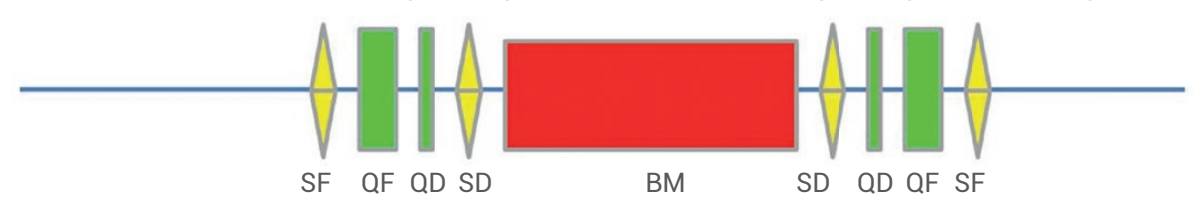

2. Une des seize cellules de la maille de l'anneau de stockage de SESAME.

BM : dipôle combiné. D1... D6 : distances.

QF et QD : quadrupôles respectivement focalisant et défocalisant.

SF et SD : sextupôles respectivement focalisant et défocalisant.
(Amman, octobre 2002) qui a exprimé la nécessité d'accroitre l'énergie des photons jusqu'au seuil $\mathrm{K}$ du sélénium, utilisé en cristallographie des protéines $^{(b)}$. Le nouvel anneau de stockage est constitué de 16 cellules identiques. Les éléments de base de chaque cellule sont des dipôles combinés, entourés par un ensemble de quadrupôles et de sextupôles (fig. 2). La focalisation verticale est assurée par le dipôle combiné et la focalisation horizontale par les quadrupôles. La circonférence de l'anneau est portée à 130 mètres, l'émittance (produit de la taille du faisceau d'électrons par sa divergence) étant de 25 nm.rad à 2,5 GeV. Les spécifications de la machine sont résumées dans le Conceptual Design Report consigné dans le SESAME Yellow Book [5].

\section{Infrastructures}

La construction du bâtiment a débuté en 2003 et l'emménagement à l'intérieur des nouveaux locaux en 2008. Les dimensions du hall expérimental $\left(60 \times 60 \mathrm{~m}^{2}\right)$ ont été choisies pour héberger l'anneau de $130 \mathrm{~m}$ de circonférence et disposer de lignes de lumière de $30 \mathrm{~m}$ de longueur. Les trois accélérateurs (microtron, booster et anneau de stockage) sont logés dans des tunnels en béton dont les caractéristiques ont été déterminées par un calcul de radioprotection validé par des experts internationaux. Le bâtiment principal, conçu pour absorber les vibrations verticales, comprend les tunnels hébergeant les trois accélérateurs, le hall expérimental avec les lignes de lumière, les laboratoires et les bureaux. La stabilité est encore améliorée par le découplage total des fondations du hall expérimental de celles du reste du bâtiment.

Un bâtiment technique, séparé du bâtiment principal avec lequel il n'est relié que par un pont couvert assurant le passage des câbles et des canalisations de fluides, abrite tous les équipements de refroidissement et une partie des transformateurs. Ainsi, les vibrations générées par le fonctionnement des composants électromécaniques ne sont pas transférées au bâtiment principal.

Une station électrique avec quatre transformateurs, connectée à la Compagnie électrique jordanienne par un câble dédié de 7,2 km de long, assure une puissance de 6 MVA. Elle est complétée depuis début 2019 par une centrale solaire qui fournira l'essentiel de l'énergie électrique, faisant de SESAME la première source synchrotron alimentée en énergie renouvelable. 


\section{Mise en opération du microtron et du booster}

Le premier faisceau d'électrons du microtron a été obtenu le 14 juillet 2009 à une énergie de $9,1 \mathrm{MeV}$, permettant d'effectuer les tests préliminaires. Il a été extrait à l'énergie maximale de $22,5 \mathrm{MeV}$ en novembre 2011, après la construction du mur de blindage définitif. Les autres paramètres du faisceau sont proches des valeurs prévues. Plusieurs tests ont montré la bonne reproductibilité du faisceau et la fiabilité des équipements. C'est une belle réussite, récompensant le grand effort du personnel de SESAME.

Le booster de SESAME a été construit à partir d'éléments de BESSY I. Avant sa mise en opération avec faisceau, plusieurs tests ont été effectués et plusieurs améliorations réalisées. Tous les aimants (12 dipôles, 12 quadrupôles focalisants et 6 quadrupôles défocalisants) ont été testés électriquement et hydrauliquement. Le système d'alignement a été amélioré pour les dipôles et construit pour les quadrupôles. Les alimentations électriques ont été remplacées. Les aimants pulsés [6], le système radiofréquence (RF) et leurs alimentations ont été testés. Le système de vide et une partie du système de contrôle ont été renouvelés. De nouveaux diagnostics, par exemple sept nouveaux modules de lecture locale de la position du faisceau, ont été introduits.

Le 3 septembre 2014, l'équipe de SESAME a réussi à accélérer à $800 \mathrm{MeV}$ les électrons dans le booster et à valider son fonctionnement aux valeurs nominales. L'émittance mesurée, $120 \mathrm{~nm}$.rad, est proche de la valeur théorique, avec un courant maximum accéléré de l'ordre de $5 \mathrm{~mA}$, voisin de sa valeur dans BESSY I.

À la sortie du booster, les électrons passent à travers une ligne de transfert vers l'anneau de stockage qui, à chaque remplissage, maintient un faisceau d'électrons de $200 \mathrm{~mA}$ (400 mA à terme) à $2,5 \mathrm{GeV}$, circulant pendant plusieurs heures.

\section{Construction, montage et mise en opération de l'anneau de stockage}

L'anneau de stockage est complètement nouveau. Sa conception et l'évaluation de ses couts ont été finalisées en 2009 sous la

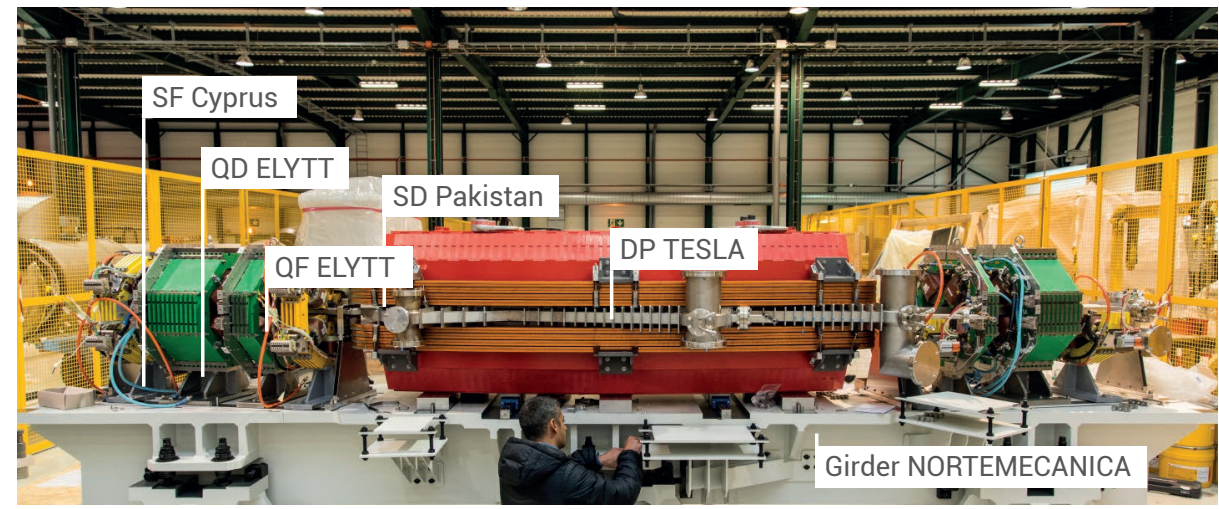

3. Une des seize cellules de l'anneau de stockage de SESAME, assemblée au CERN avant son expédition à Amman. Les noms des entreprises ou des pays ayant contribué à la construction des différents éléments sont indiqués.

direction d'Amor Nadji et validées par le Comité consultatif technique international de SESAME (TAC), regroupant des experts des laboratoires mondiaux de rayonnement synchrotron.

Les éléments magnétiques ont été construits dans le cadre du contrat européen (FP7) CESSAMag [7]. L'Union européenne a financé le cout des aimants et des poutres-supports (girders), et le CERN a mis à disposition ses experts pour le suivi de la construction à laquelle des entreprises de pays membres de SESAME (Chypre, Pakistan et Turquie) ont contribué, comme précisé sur la figure 3. Ces entreprises, qui n'avaient aucune expérience dans la production de composants d'accélérateur, avaient le potentiel de le faire, d'après une évaluation d'experts du CERN. En passant la commande d'un prototype, le CERN a pu tester les composants et s'assurer qu'ils étaient fabriqués selon les spécifications de SESAME. De fait, la société turque Elytt a construit les bobines des quadrupôles, et les sextupôles (66 aimants) ont été assemblés moitié par une société chypriote et moitié par une société pakistanaise, leurs alimentations ayant été acquises auprès d'une compagnie israélienne.

Le système radiofréquence (RF) de l'anneau de stockage de SESAME est composé de quatre cavités accélératrices à $500 \mathrm{MHz}$ de type ELETTRA (centre synchrotron italien), alimentées par quatre amplificateurs à semi-conducteurs de $80 \mathrm{~kW}$ chacun. Le premier a été conçu et construit à SOLEIL et les trois autres fournis par l'entreprise française SIGMAPHI ELECTRONICS, qui détient une licence exclusive d'exploitation de la technologie SOLEIL.
L'installation de l'anneau de stockage a été achevée en novembre 2016 sous la direction d'Erhard Huttel. La figure 4 montre une partie de l'anneau où l'on peut apercevoir les aimants, une partie de la chambre à vide et les chemins de câbles. Le premier faisceau d'électrons a circulé le 12 janvier 2017. Les physiciens des accélérateurs de SESAME ont ensuite successivement réussi à lui faire faire plusieurs tours, puis à stocker et enfin accumuler un faisceau dans l'anneau de stockage après des réglages délicats d'orbite verticale. L'énergie nominale de $2,5 \mathrm{GeV}$ a été atteinte en avril 2017. Le temps nécessaire pour passer de l'energie d'injection de $800 \mathrm{MeV}$ à l'énergie de travail de l'anneau de stockage de 2,5 GeV est de l'ordre de 190 secondes. L'optimisation du fonctionnement de l'anneau se poursuit avec des résultats prometteurs. En novembre 2018, le courant dans l'anneau avait atteint $200 \mathrm{~mA}$ avec une durée de vie de l'ordre de 15 heures.

\section{Les lignes de lumière de la phase 1}

La sélection des lignes de lumière de la phase 1 a été basée sur les contributions d'au moins cinq ateliers et des réunions d'utilisateurs, auxquelles ont participé plusieurs centaines de scientifiques du Moyen-Orient. Parmi les sept lignes retenues, trois sont opérationnelles, une est en construction et deux en conception. La ligne XAFS/XRF (structure fine d'absorption des rayons $\mathrm{X}$ et fluorescence $\mathrm{X}$, $4,5$ à $30 \mathrm{keV})$, donation de l'institut HZDR (Dresde, Allemagne), installée sur un aimant de courbure, est en exploitation sous la responsabilité de Messaoud 


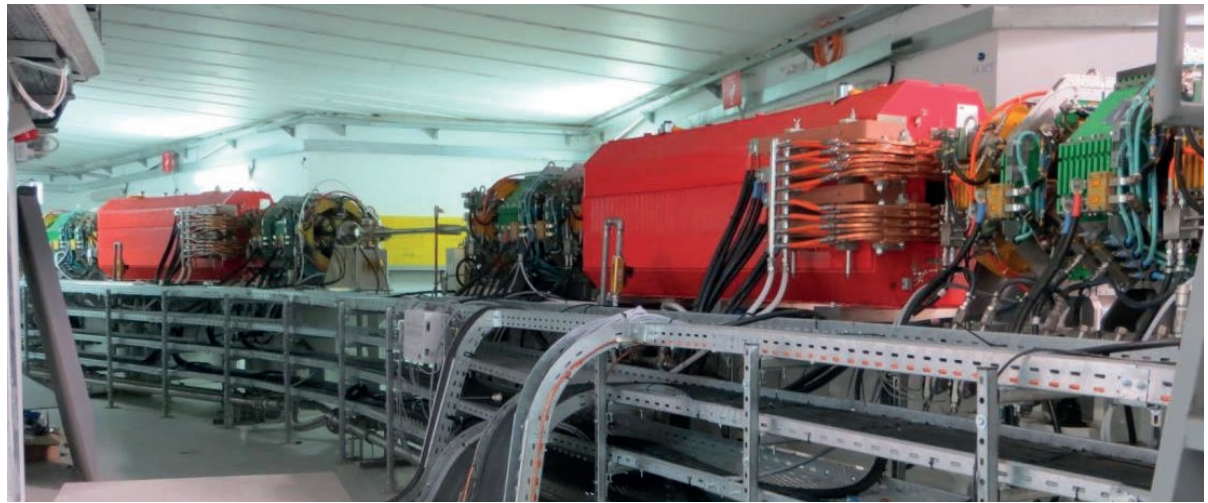

4. Vue d'une partie de l'anneau de stockage dans son tunnel.

Harfouche. Elle a fourni son premier spectre au seuil d'absorption $\mathrm{K}$ du cuivre le 22 novembre 2017 (fig. 5). Elle a accueilli en juillet 2018 ses premiers utilisateurs : une équipe de paléontologues du Cyprus Institute, animée par la professeure Kirsi Lorentz, dont le projet était l'un des dix-neuf retenus sur les cinquanteneuf déposés lors du premier appel. L'équipe a détecté et dosé des éléments métalliques sur des restes d'ossements humains de la région, datant du Néolithique. La ligne XAFS/XRF a publié son premier article sur la caractérisation de catalyseurs de reformage de $\mathrm{CO}_{2}$ [8].

La seconde ligne de lumière (IR) est une ligne de spectromicroscopie infrarouge $(0,1$ à $3 \mathrm{eV})$, utilisant un des aimants de courbure de la machine, conçue et construite en étroite collaboration avec SOLEIL sous la responsabilité de Gihan Kamal (SESAME) et Paul Dumas (SOLEIL). Elle est opérationnelle depuis septembre 2018 (fig. 6) et a accueilli plusieurs équipes extérieures, dont celle du professeur Gehan Ahmad du National Research Center d'Egypte, qui a présenté au dernier Conseil SESAME des résultats remarquables sur la maladie d'Alzheimer. Le premier article issu de cette ligne [9] porte sur les résultats obtenus par une équipe jordanienne regroupant des chercheurs en pharmacologie et en médecine, sur la biochimie du placenta et du plasma de pré-éclampsie, syndrome affectant des femmes enceintes.

La troisième ligne de lumière (MS), installée sur un wiggler, est en test sous faisceau. Transférée du centre synchrotron suisse SLS et équipée d'un nouveau détecteur très performant, elle est dediée à la diffraction des rayons $\mathrm{X}$ (entre 5 et $25 \mathrm{keV}$ ) sur poudres en science des matériaux. Elle a reçu son premier faisceau le 23 décembre 2019 et accueillera ses premiers utilisateurs fin 2020 .

Une quatrième ligne (MX), prévue pour la cristallographie des macromolécules, est en cours de construction ; c'est une ligne de lumière complètement nouvelle, dont lses photons seront issus d'un onduleur sous vide.

Enfin, deux autres lignes sont lancées : une ligne de tomographie X (BEAMS), financée par l'Europe à hauteur de $6 \mathrm{M} €$, et une ligne de spectroscopie de photoémission dans le domaine des rayons $\mathrm{X}$ mous, cofinancée par l'association Helmholtz allemande et le commissariat à l'énergie atomique de Turquie (TAEC).

\section{La formation}

Un comité international, regroupant des experts dans le domaine des accélérateurs, a contribué à organiser efficacement la formation en liaison avec les installations internationales. Le premier plan de formation a été consacré aux experts machine destinés à concevoir et construire l'accélérateur.

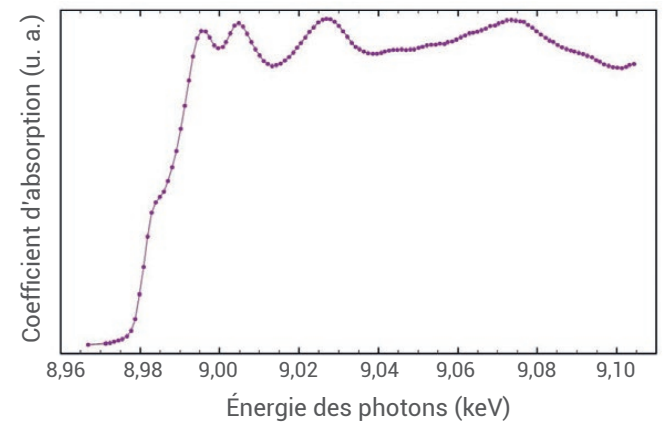

5. Spectre XANES au seuil $K$ du cuivre, obtenu le 4 décembre 2017 sur la ligne de lumière XAFS/XRF.

(Courtoisie du Dr. M. Harfouche, SESAME). financement, notamment par l'intégration
Une centaine de candidats de la région ont participé à une école de deux semaines dédiée aux accélérateurs de particules et aux technologies associées. À l'issue de cette école, 20 à 25 participants ont été sélectionnés pour une formation spécifique de longue durée ( 18 mois) proposée individuellement dans l'un des laboratoires européens du domaine. Ils ont pu y approfondir leurs connaissances en participant à des écoles très réputées comme le CAS (Cern Accelerator School). À la suite de cette formation longue, plusieurs participants ont rejoint le projet SESAME et contribué de façon significative à sa construction.

\section{Le financement du projet}

En 2017, le cout total du projet avait atteint 90 millions de dollars. Le financement de SESAME a été réparti entre les États membres et le soutien international : - Site, bâtiments et infrastructures (routes, électricité et eau) payés par la Jordanie $(12,77 \mathrm{M} \$)$; centrale électrique solaire financée avec l'aide de l'Union européenne à la Jordanie $(7 \mathrm{M} \$)$.

- Budget annuel d'exploitation (salaires, électricité, eau, différents consommables...) financé par les actuels États membres. Ce budget, décidé annuellement par le Conseil, s'est élevé à 5,288 M\$ en 2018 et est appelé à croitre avec l'augmentation du nombre de lignes expérimentales et d'utilisateurs. L'enjeu est de consolider ce de nouveaux États membres.

- Système d'injection offert par l'Allemagne et mis à niveau par SESAME (10 M\$), anneau de stockage (29,68 M\$) financé par l'Union européenne (11,3 M\$) et trois pays membres de SESAME (Israël, Jordanie et Turquie). 
- Lignes de lumière, dont certaines sont des donations d'autres laboratoires (23,3 M\$).

- Formation de scientifiques, ingénieurs et techniciens sur des budgets financés par l'AIEA, des sociétés savantes, des sociétés caritatives, et l'Union européenne via le programme OPEN SESAME (voir ci-après).

\section{Conclusion et perspectives}

SESAME vient de basculer de sa phase de construction à celle d'exploitation. La machine délivre déjà un faisceau de $200 \mathrm{~mA}$ à $2,5 \mathrm{GeV}$, d'une durée de vie suffisante pour réaliser des expériences de niveau international. Le personnel technique, scientifique et administratif de SESAME est composé aujourd'hui de 48 personnes. Onze recrutements sont programmés en 2020 grâce à la réduction de 90\% du budget annuel d'électricité générée par la centrale solaire. Suite au premier appel, 103 projets ont été déposés par des équipes d'une dizaine de pays.

Les premiers utilisateurs, dont les projets ont été sélectionnés par un comité d'experts indépendants, ont été accueillis dès l'été 2018. Entre juillet et décembre 2018, 23 expériences ont ainsi été réalisées sur les deux lignes de lumière XAFS/XRS et IR, par des équipes provenant de différents pays (Chypre, Turquie, Égypte, Iran, Pakistan, Jordanie) et de différentes disciplines (archéologie, sciences de la vie, chimie des batteries et de l'environnement, sciences des matériaux). 45 expériences ont été réalisées en 2019. Quatre articles ont été publiés ou sont en cours de publication dans des revues internationales. C'est un succès remarquable, qui montre que SESAME est déjà une institution structurante pour la recherche et l'innovation dans la région, comme en témoignent les 151 demandes de faisceau déposées à la suite du second appel à projets de janvier 2020. Cette dynamique va encore s'accélérer avec la mise en service en 2020 de la troisième ligne expérimentale de diffraction $\mathrm{X}$ (MS) dédiée aux sciences des matériaux. La maison d'hôtes, financée par l'Italie et inaugurée en décembre 2019, accueille les utilisateurs dans ses 45 chambres en partie meublées par la Suisse ; elle dispose d'un service de restauration et de salles de travail et de conférences très fonctionnelles, permettant ainsi à SESAME d'œuvrer en continu, y compris les week-ends.

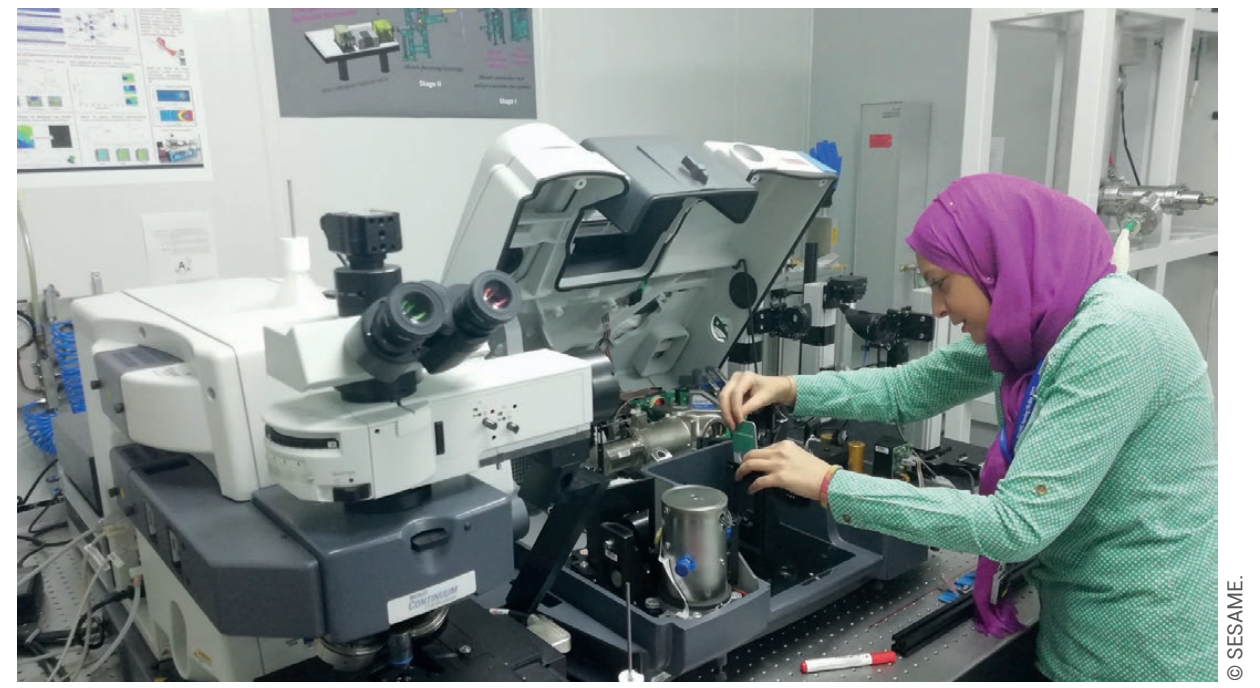

6. Mise en place d'une expérience sur la ligne de lumière IR (spectromicroscopie infrarouge) du synchrotron SESAME.

SESAME est déjà impliqué dans plusieurs collaborations avec les autres centres de rayonnement synchrotron et dans des programmes européens tel que OPEN SESAME, destiné à insérer ses utilisateurs dans les principaux axes de recherche utilisant le rayonnement synchrotron. SESAME est aussi associé depuis novembre 2018 à la Ligue européenne des sources de photons (LEAPS) basées sur des accélérateurs.

L'enjeu reste toutefois d'assurer et de sécuriser le budget d'exploitation du centre et d'amener les pays membres à garantir un financement régulier, à la hauteur de la mission. Cet enjeu est d'autant plus important que le nombre de lignes de lumière va augmenter de trois à six dans les prochaines années (voir ci-dessus). Par ailleurs, des mises à niveau sont envisagées, notamment le remplacement du microtron par un nouvel injecteur.

Le pari est donc tenu et toutes les conditions sont remplies pour offrir aux scientifiques de la région et d'ailleurs un centre d'excellence ouvert, multi et interdisciplinaire, qui stimulera des recherches de haut niveau, des collaborations et des échanges fructueux, générateurs de résultats scientifiques, technologiques et culturels bénéfiques pour la région et ses habitants. La vitalité de SESAME est la preuve qu'il est possible de réussir la gageure de faire travailler ensemble des pays constamment en très forte tension politique. C'est bien là un des grands mérites de la pratique de la science.

\section{Références}

1 • « La lumière synchrotron au service de la science et de la société ", Reflets de la physique, numéro spécial 34-35 (2013).

2. www.synchrotron-soleil.fr

3. A. Michalowicz et al., « De Frascati à Soleil, histoire du rayonnement synchrotron (1963-2013) », Histoire de la recherche contemporaine, tome III-1 (2014), http://journals. openedition.org/hrc/413; DOI : $10.4000 /$ hrc. 413

4. Sesame White Book (juillet 2002), https://www.sesame.org.jo

5. Sesame Yellow Book (mai 2003), https://www.sesame.org.jo

6. S. Varnasseri et al., "Finalized design of the pulsed magnets and their PS for SESAME ring injection", Proceedings of EPAC08, Genoa, Italy (23-27 juin 2008).

7• https://cessamag.web.cern.ch/

8. S. Bac et al., "Exceptionally active and stable catalysts for $\mathrm{CO}_{2}$ reforming of glycerol to syngas", Applied Catalysis B: Environmental, 256 (2019) 117808.

9• L.A. Dahabiyeh et al., "Investigating the molecular structure of placenta and plasma in pre-eclampsia by infrared microscopy", J. Pharm. Biomed. Anal., 184 (2020) 113186.

(a) Nous souhaitons rendre hommage à Michel Sommer, ingénieur et alors directeur des anneaux du Lure, pour son engagement enthousiaste dans le projet et le rôle qu'il a joué dans la formation et la sélection des premiers ingénieurs machine de SESAME.

(b) En effet, la substitution des atomes de soufre des méthionines et des cystéines par des atomes de sélénium permet d'obtenir des informations sur la phase du signal diffracté et de résoudre la structure de la protéine. 